\title{
Ghosts of Milky Way's past: the globular cluster ESO 37-1 (E 3) ${ }^{\star, \star \star, \star \star \star}$ (Research Note)
}

\author{
R. de la Fuente Marcos ${ }^{1}$, C. de la Fuente $\operatorname{Marcos}^{1}$, C. Moni Bidin ${ }^{2}$, S. Ortolani ${ }^{3}$, and G. Carraro ${ }^{4,3}$ \\ 1 Apartado de Correos 3413, 28080 Madrid, Spain \\ e-mail: rauldelafuentemarcos@gmail.com \\ ${ }^{2}$ Instituto de Astronomía, Universidad Católica del Norte, Av. Angamos 0610, Antofagasta, Chile \\ 3 Dipartimento di Fisica e Astronomia, Università degli Studi di Padova, Vicolo dell'Osservatorio 3, 35122 Padova, Italy \\ ${ }^{4}$ European Southern Observatory, Alonso de Cordova 3107, Casilla 19001, Santiago 19, Chile
}

Received 22 May 2015 / Accepted 5 July 2015

\section{ABSTRACT}

\begin{abstract}
Context. In the Milky Way, most globular clusters are highly conspicuous objects that were found centuries ago. However, a few dozen of them are faint, sparsely populated systems that were identified largely during the second half of the past century. One of the faintest is ESO 37-1 (E 3) and as such it remains poorly studied, with no spectroscopic observations published so far although it was discovered in 1976.

Aims. We investigate the globular cluster E 3 in an attempt to better constrain its fundamental parameters. Spectroscopy of stars in the field of $\mathrm{E} 3$ is shown here for the first time.

Methods. Deep, precise VI CCD photometry of E 3 down to $V \sim 26$ mag is presented and analysed. Low-resolution, medium signalto-noise ratio spectra of nine candidate members are studied to derive radial velocity and metallicity. Proper motions from the UCAC4 catalogue are used to explore the kinematics of the bright members of E3.

Results. Isochrone fitting indicates that E 3 is probably very old, with an age of about 13 Gyr; its distance from the Sun is nearly $10 \mathrm{kpc}$. It is also somewhat metal rich with $[\mathrm{Fe} / \mathrm{H}]=-0.7$. Regarding its kinematics, our tentative estimate for the proper motions is $\left(\mu_{\alpha} \cos \delta, \mu_{\delta}\right)=(-7.0 \pm 0.8,3.5 \pm 0.3)$ mas $\mathrm{yr}^{-1}$ (or a tangential velocity of $382 \pm 79 \mathrm{~km} \mathrm{~s}^{-1}$ ) and for the radial velocity $45 \pm 5 \mathrm{~km} \mathrm{~s}^{-1}$ in the solar rest frame.

Conclusions. E 3 is one of the most intriguing globular clusters in the Galaxy. Having an old age and being metal rich is clearly a peculiar combination, only seen in a handful of objects like the far more conspicuous NGC 104 (47 Tucanae). In addition, its low luminosity and sparse population make it a unique template for the study of the final evolutionary phases in the life of a star cluster. Unfortunately, E 3 is among the most elusive and challenging known globular clusters because field contamination severely hampers spectroscopic studies.
\end{abstract}

Key words. globular clusters: general - globular clusters: individual: ESO 37-1 - Galaxy: halo - Galaxy: structure - Local Group

\section{Introduction}

In the Milky Way, most globular clusters are highly conspicuous and rich stellar systems that were discovered centuries ago (see e.g. Hanes 1980; Ashman \& Zepf 2008). However, a few dozen objects are faint and sparsely populated; they were identified predominantly during the second half of the past century (see e.g. Harris 1976, 1996). Most globular clusters were born with the Galaxy, but others were captured and today are found associated with more or less prominent stellar streams (see e.g.

\footnotetext{
* This research note is based on observations made with the ESO VLT at the Paranal Observatory, under the program 078.D-0186 and includes data gathered with the $6.5 \mathrm{~m}$ Magellan Telescopes located at Las Campanas Observatory, Chile (program ID CHILE-2015A-029).

$\star \star$ Figure 6 and Appendix A are available in electronic form at http://www. aanda.org

$\star \star \star$ Tables of the individual photometric measurements are only available at the CDS via anonymous ftp to cdsarc.u-strasbg. fr $(130.79 .128 .5)$ or via

http://cdsarc.u-strasbg.fr/viz-bin/qcat?]/A+A/581/A13
}

Lynden-Bell \& Lynden-Bell 1995; Freeman \& Bland-Hawthorn 2002; Forbes \& Bridges 2010).

One of the faintest known globular clusters is ESO 37-1 (E 3), which was discovered by A. Lauberts in Chamaeleon in 1976 (Lauberts 1976); it is still a poorly studied object and no spectroscopic observations have been published. Harris (2010) gives the position of the cluster as $\alpha(\mathrm{h}: \mathrm{m}: \mathrm{s}, \mathrm{J} 2000)=9: 20: 57.07$, $\delta\left({ }^{\circ}::^{\prime \prime}, \mathbf{J} 2000\right)=-77: 16: 54.8$ or $l=292^{\circ} .270, b=-19.020$, with $M_{V}=-4.12 \mathrm{mag},[\mathrm{Fe} / \mathrm{H}]=-0.83$ dex and located at a heliocentric distance of $8.1 \mathrm{kpc}$ or galactocentric distance of $9.1 \mathrm{kpc}$. Sarajedini et al. (2007) point out that E3 appears to be $\sim 2$ Gyr younger than NGC 104 (47 Tucanae), a very conspicuous $12 \pm 1$ Gyr old (McDonald et al. 2011) globular cluster.

Here, we present and analyse deep, precise VI CCD photometry of $\mathrm{E} 3$ down to $V \sim 26 \mathrm{mag}$. In addition, we study low-resolution, medium signal-to-noise ratio spectra of nine candidate cluster members. This research note is organised as follows. Section 2 is devoted to photometry. The spectroscopy is discussed in Section 3. The proper motions of possible bright members of the cluster are investigated in Sect. 4. In Sect. 5, a 


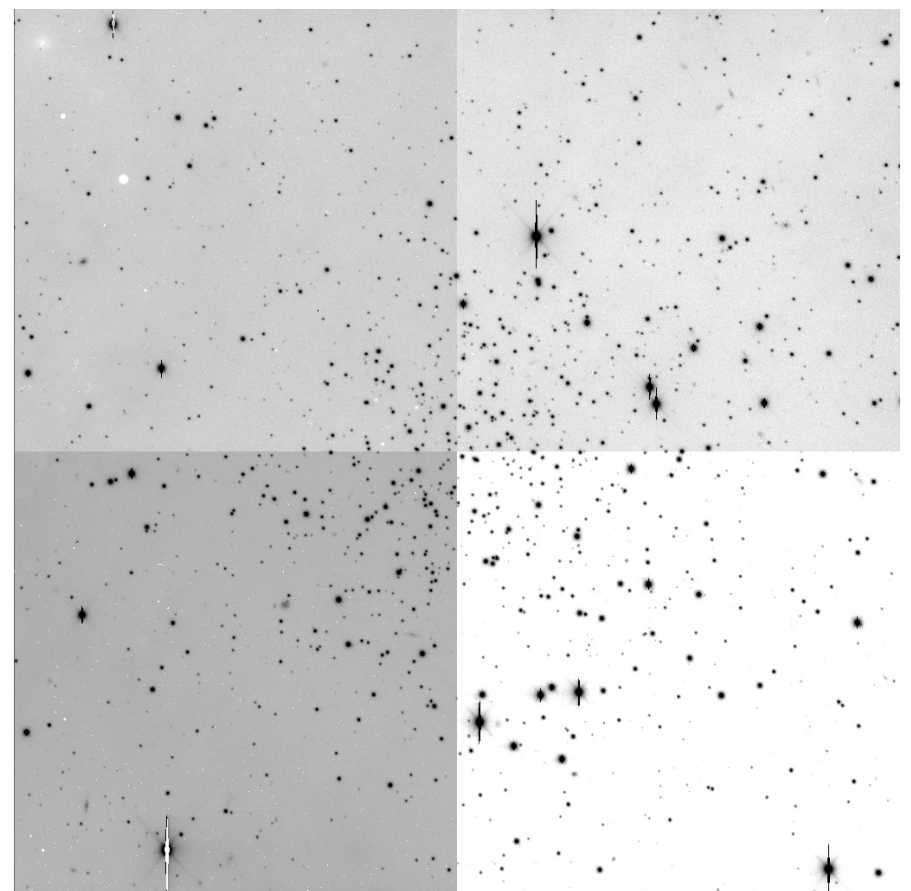

Fig. 1. $V$ 1000-s exposure in the field of E 3. North is up, east to the left, and the field is 6.8 on a side.

polar path analysis is presented. Our results are discussed and our conclusions summarised in Sect. 6.

\section{Photometry}

On the night of 13 November 2006, E 3 was observed in the $V I$ filter bands with the Very Large Telescope UT2 Kueyen and the FORS1 CCD camera. Four exposures were taken $(10 \mathrm{~s}$ and $1000 \mathrm{~s}$ in both $V$ and $I$ ) on that night, which had nominal photometric conditions and an average seeing of 0.8 , at an airmass in the range $1.77-1.82$. The camera has a scale of 0.2 per pixel and an array of $2048 \times 2048$ pixels. An example of one of the $6.8 \times 6.8 \operatorname{arcmin}^{2}$ images of E 3 is shown in Fig. 1. An adjacent field was observed under the same conditions, offset by half a degree in the west direction. The standard IRAF ${ }^{1}$ routines were applied to reduce the raw images. Using the psf-fitting routines of DAOPHOT and ALLSTAR (Stetson 1994) in the IRAF environment, we measured instrumental magnitudes for all the stars in the field. These magnitudes were converted to the standard system using the stars in common with Veronesi et al. (1996) as local standards; transformations were subsequently utilised to tie both the cluster and the field photometry to them (tables including the individual photometric measurements are available at CDS).

The resulting colour magnitude diagrams (CMD) are shown in Fig. 2, for both the cluster (left panel) and the field (right panel). One can readily see that we can cover the cluster main sequence (MS) from the turn-off point (TO) down to $V \sim 25 \mathrm{mag}$, two magnitudes fainter than Veronesi et al. (1996). The photometry reaches approximately the same depth as the archival HST images of E 3 discussed in Lan et al. (2010). For the purpose of this study, we combined our photometry with that in

\footnotetext{
1 IRAF is distributed by the National Optical Astronomy Observatory, which is operated by the Association of Universities for Research in Astronomy (AURA) under a cooperative agreement with the National Science Foundation.
}

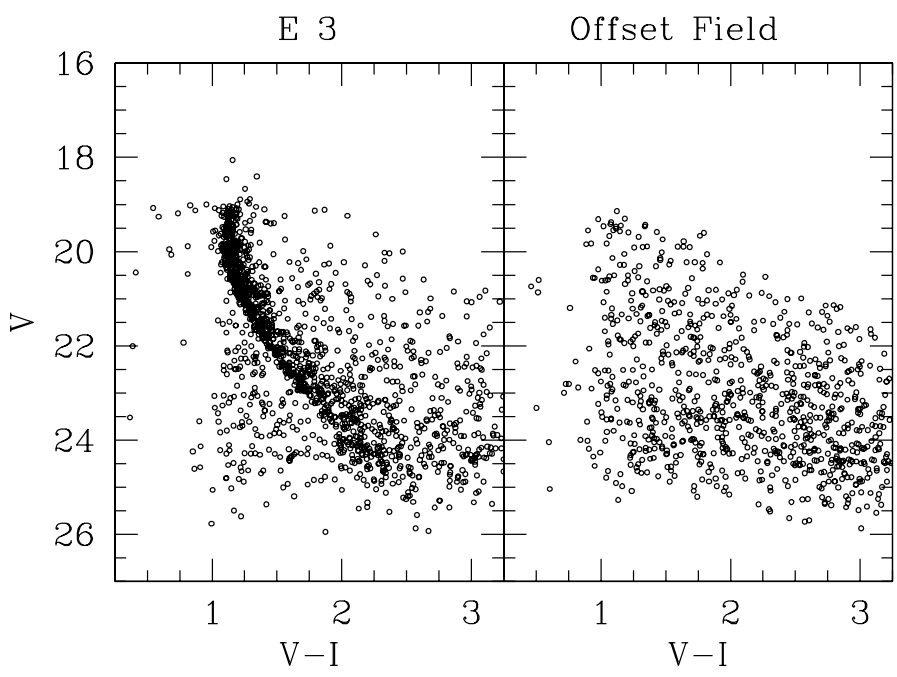

Fig. 2. CMD for E3 (left panel) and the comparison field (right panel).

Table 1. Results of the completeness test.

\begin{tabular}{ccc}
\hline \hline & Cluster & Field \\
\hline $\begin{array}{c}V \\
(\mathrm{mag})\end{array}$ & $\begin{array}{c}\text { comp } \\
(\%)\end{array}$ & $\begin{array}{c}\text { comp } \\
(\%)\end{array}$ \\
\hline $20.0-23.0$ & 100 & 100 \\
$23.5-24.5$ & 90 & 100 \\
$24.5-25.0$ & 64 & 90 \\
$25.0-25.5$ & 17 & 55 \\
$\geq 25.5$ & $\geq 0$ & 20 \\
\hline$I$ & comp & comp \\
$(\mathrm{mag})$ & $(\%)$ & $(\%)$ \\
\hline $20.0-22.7$ & 100 & 100 \\
$22.7-24.0$ & 72 & 100 \\
$23.0-24.5$ & 24 & 68 \\
$24.5-25.0$ & 10 & 32 \\
\hline
\end{tabular}

Gratton \& Ortolani (1987) to have a complete magnitude coverage, and to also sample the evolved part of the CMD (see below). Photometric errors for stars with $V \approx 20 \mathrm{mag}$ are $\sim 0.03 \mathrm{mag}$ $(0.05$ in $V-I)$; for $V \approx 23$ mag they amount to $\sim 0.10$ mag $(0.15$ in $V-I)$.

In order to estimate star membership in the cluster, we made use of both the photometry of the cluster and the accompanying offset field. First of all, we performed a completeness analysis, both for the cluster and for the field, which is summarised in Table 1. This analysis was carried out in the standard way (see Baume et al. 2007) by adding a number of stars per different magnitude bin and randomly across the field, and then computing the ratio of recovered to injected stars, where the recovered stars result from the reduction of the added-star-image under the same conditions as in the original image. Looking at Table 1, one can conclude that our photometry is complete down to $V \sim 25 \mathrm{mag}(I \sim 24 \mathrm{mag})$ and that the cluster is affected by some crowding.

The completeness results have been subsequently applied to perform a statistical cleaning of the cluster CMD making use of the field, as described in Carraro \& Costa (2007), with the aim of isolating statistically the genuine cluster population. Summarising, the statistical technique we employ works as follows. We pick an offset field star and look into the cluster CMD for the closest star in colour and magnitude using a search ellipse, and we remove this star from the cluster star list. This 


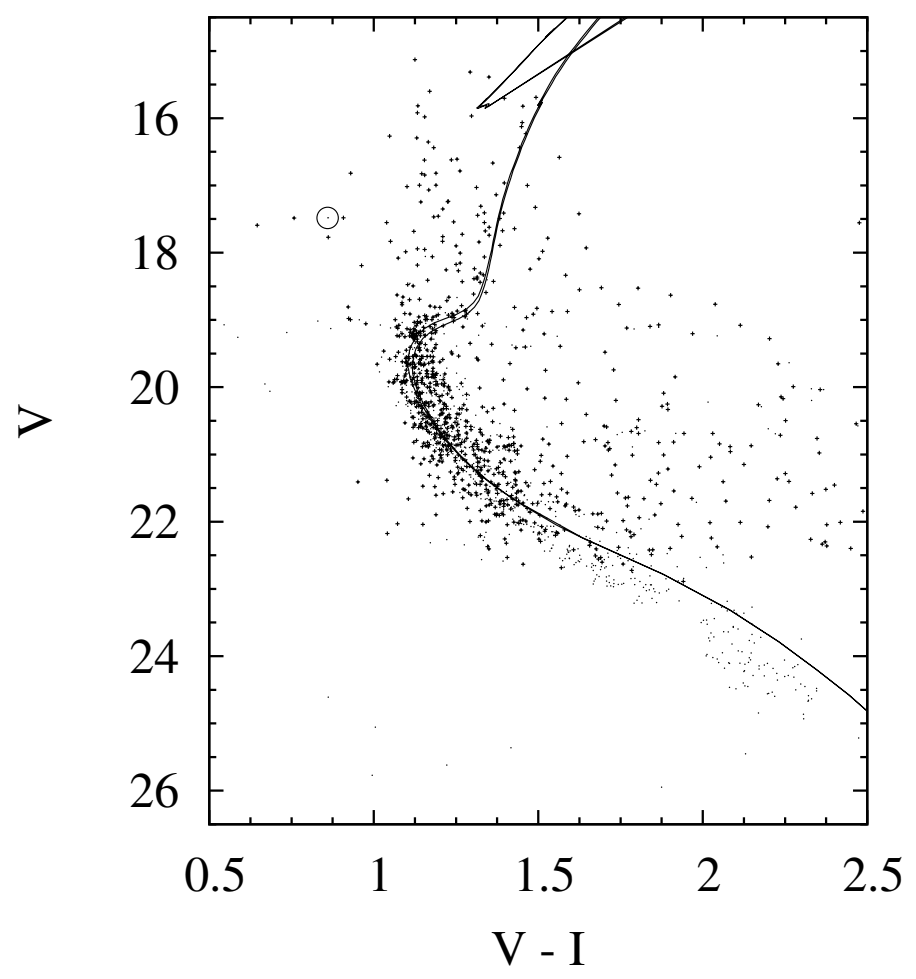

Fig. 3. CMD for the clean sample (points). Photometry from Gratton \& Ortolani (1987) is also shown (crosses). Two isochrones (12-13 Gyr, $Z=0.003$ ) from the PARSEC evolutionary tracks (Bressan et al. 2012) are shown. The blue straggler V1, an SX Phoenicis variable star (Mochejska et al. 2000) and a probable cluster member, is shown as a large empty circle.

procedure is repeated for all the stars in the offset field. We refer the reader to the cited paper for the full details on this procedure. In the following analysis we make use of this resulting clean CMD.

Figure 3 shows the CMD for the clean sample as black circles, and data from Gratton \& Ortolani (1987) as red circles. Two isochrones with $Z=0.003$, and 12 and $13 \mathrm{Gyr}$ of age are used to perform a main-sequence fitting, giving $\left(V-M_{V}\right)_{0}=15.07 \mathrm{mag}$ and $E(V-I)=0.47$; the values of $A_{V}$ and $A_{I}$ from Schlafly $\&$ Finkbeiner (2011) are $0.93 \mathrm{mag}$ and $0.51 \mathrm{mag}$, respectively. Using these values, our estimate for the cluster heliocentric distance is $10.3 \pm 0.9 \mathrm{kpc}$. As the studied field is restricted to 6.8 on a side, we disregard estimating the cluster absolute magnitude and structural parameters from the photometry presented here.

\section{Spectroscopy}

Intermediate-resolution $(R \approx 7000)$ spectra of nine stars in the field of E3 were collected at the $6.5 \mathrm{~m}$ Baade Telescope, Las Campanas Observatory, on 28-29 January and 22 February 2015. The IMACS spectrograph was used at $\mathrm{f} / 4$, with the $1200+26.7$ grating and 0 ! 75 -wide slit. The resulting spectral range 5200-7000 $\AA$ was covered by four chips, with one short gap between each pair of chips. Exposure times varied between $240 \mathrm{~s}$ and $900 \mathrm{~s}$, depending on target magnitude. The four spectral sections were reduced individually with standard IRAF routines, extracted and normalised. Before merging them, the offsets between the four independent wavelength calibration solutions were measured on a twilight solar spectrum and corrected. We measured the radial velocity (RV) of the target stars cross-correlating (see Tonry \& Davis 1979) their spectra
Table 2. Spectroscopic results.

\begin{tabular}{c|ccrcll}
\hline \hline ID & $\begin{array}{c}\text { RA(J2000) } \\
(\text { h:m:s })\end{array}$ & $\begin{array}{c}\text { Dec(J2000) } \\
\left({ }^{\prime}::^{\prime \prime}\right)\end{array}$ & $\begin{array}{r}\text { RV } \\
\left(\mathrm{km} \mathrm{s}^{-1}\right)\end{array}$ & $\begin{array}{c}T_{\text {eff }} \\
(\mathrm{K})\end{array}$ & $\begin{array}{c}V \\
(\mathrm{mag})\end{array}$ & Notes \\
\hline 1 & $09: 20: 48.0$ & $-77: 15: 15$ & $6 \pm 9$ & 5500 & 13.84 & field (hot) \\
2 & $09: 20: 55.9$ & $-77: 19: 01$ & $-23 \pm 3$ & 6800 & 14.55 & field (hot) \\
3 & $09: 20: 41.9$ & $-77: 18: 47$ & $-35 \pm 3$ & 5750 & 14.55 & field (hot) \\
4 & $09: 20: 32.1$ & $-77: 16: 25$ & $-21 \pm 4$ & 4900 & 14.58 & field (met.) \\
5 & $09: 20: 47.3$ & $-77: 18: 48$ & $6 \pm 4$ & 4600 & 14.74 & field (met.) \\
6 & $09: 20: 31.2$ & $-77: 16: 33$ & $45 \pm 3$ & 4950 & 14.84 & Member \\
15 & $09: 19: 23.5$ & $-77: 14: 39$ & $44 \pm 5$ & 4650 & 15.59 & Member \\
17 & $09: 19: 41.6$ & $-77: 08: 07$ & $41 \pm 4$ & 5600 & 15.64 & field \\
18 & $09: 19: 17.2$ & $-77: 09: 40$ & $28 \pm 6$ & 5700 & 16.03 & field (hot) \\
\hline
\end{tabular}

with synthetic templates drawn from the library of Coelho et al. (2005). The results were corrected to heliocentric velocities and for zero-point offsets, estimated from the position of the strong telluric band observed at 6850-7000 $\AA$. The errors were computed as the quadratic sum of the most relevant sources of uncertainty, namely the cross-correlation, the wavelength calibration, and the RV zero-point correction (for further details, see Carraro et al. 2007). We estimated the temperature of the targets fitting the wings of the $\mathrm{H}_{\alpha}$ line with the same synthetic spectra (see Fuhrmann et al. 1994) following the procedure applied in Moni Bidin et al. (2010). We varied the gravity and metallicity of the template models and we thus verified that, as expected, the results were largely insensitive to these parameters. The uncertainty associated with the adopted $\chi^{2}$ minimisation is likely an underestimate of the true errors because it takes into account only random errors (spectral noise), while sources of systematics such as continuum definition and selection of data points for the fit must also play a role. From the comparison of estimates obtained with different model parameters, normalisation, and fit range we estimated that the true uncertainties should likely be of the order of 150-200 K. The results are given in Table 2, while in Fig. 4 we compare the position of the stars in the temperaturemagnitude diagram with the cluster sequence. Four stars, indicated with "field (hot)" in the last column of the table, are too hot for an old stellar cluster and they will not be considered further. Unfortunately, we found no spectral feature suitable for a reliable gravity estimate at our low resolution. The degeneracy between gravity and metallicity in the fit of the spectra could therefore not be fully broken and we derived the best-fit metallicity when $\log g$ was varied in the range $0-4$ dex. The metallicity of star 4 resulted $[\mathrm{Fe} / \mathrm{H}]>-0.35$ for any value of $\log g$, and the best-fit solution of star 5 indicated that it is most likely a dwarf star $(\log g>4)$. Their cluster membership could thus be excluded and they are flagged with "field (met.)" in Table 2. The metallicity of star 17 was also too high $([\mathrm{Fe} / \mathrm{H}] \approx 0.0)$ to be of interest here. Object 6 is likely a red giant $(\log g \approx 1.8)$ with $[\mathrm{Fe} / \mathrm{H}]=-0.65 \pm 0.15$, while the spectrum of target 15 is compatible with either a metal-poor giant $(\log g \approx 2,[\mathrm{Fe} / \mathrm{H}]=$ $-0.7 \pm 0.2$ ), or a metal-rich dwarf.

In conclusion, only two stars in the observed sample (6 and 15) have both temperature and metallicity fully compatible with cluster membership, and their RVs differ by only $1 \mathrm{~km} \mathrm{~s}^{-1}$. Target 17 also has similar RV, but - although its higher temperature is still compatible with a horizontal branch cluster star - its high metallicity points to a field star. Therefore, our estimated value for the radial velocity of E 3 is $45 \pm 5 \mathrm{~km} \mathrm{~s}^{-1}$ in the solar rest frame. 


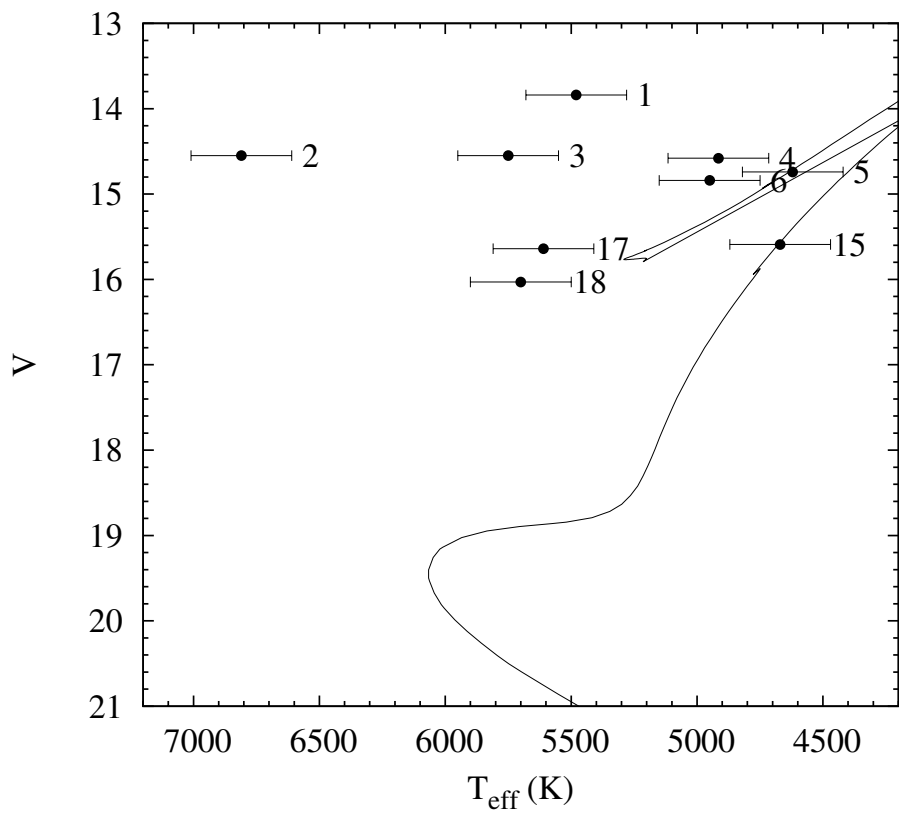

Fig. 4. Comparison between the position of the stars studied spectroscopically (see Table 2) in the temperature-magnitude diagram with an assumed cluster sequence, $Z=0.003$ and age of $13 \mathrm{Gyr}$, from the PARSEC evolutionary tracks (Bressan et al. 2012).

\section{Proper motions}

The two most robust candidates for membership in E3 have proper motions in UCAC4 (Zacharias et al. 2013). Star 6 is UCAC4 064-008502 with $\left(\mu_{\alpha} \cos \delta, \mu_{\delta}\right)=(-6.4 \pm 1.9,3.3 \pm$

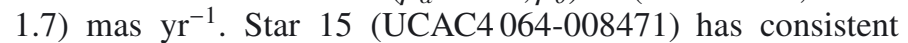
proper motions of $(-7.6 \pm 2.0,3.7 \pm 2.3)$ mas $\mathrm{yr}^{-1}$. Star 17 (UCAC4 065-008924) has somewhat inconsistent proper mo-

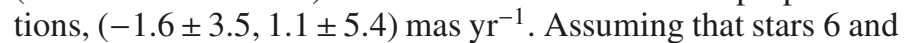
15 are part of the cluster, our tentative estimate for the proper motions is $\left(\mu_{\alpha} \cos \delta, \mu_{\delta}\right)=(-7.0 \pm 0.8,3.5 \pm 0.3) \mathrm{mas} \mathrm{yr}^{-1}$. A tangential velocity of $382 \pm 79 \mathrm{~km} \mathrm{~s}^{-1}$ is derived from the inferred cluster proper motion.

The globular cluster E 3 is a low-luminosity stellar system with very few bright stars in its central regions. The closest star to the assumed centre of the cluster (VizieR, J2000 09:20:57.1 h:m:s, -77:16:55 $\left.{ }^{\circ}:^{\prime}:^{\prime \prime}\right)$ with proper motions in UCAC4 is $40^{\prime \prime}$ from the centre. The closest putative member could be UCAC4064-008511 with $(-7.0 \pm 4.3,4.1 \pm$ 13.9) mas $\mathrm{yr}^{-1}, 63^{\prime \prime}$ from the centre. Stars 6 and 15 are located 1.4722 and 5.6358, respectively, from the centre. In contrast, star 17 is located 9.0846 from the cluster centre. Van den Bergh et al. (1980) estimated the core and tidal radii of E3 at $4.4 \mathrm{pc}$ (1.51) and $24.7 \mathrm{pc}(8.49)$, respectively. Being extra-tidal is another argument against star 17 having membership in E3.

Figure 5 shows the 2MASS near-infrared CMD of selected stars. The objects plotted have proper motions compatible with those of stars 6 (064-008502) and 15 (064-008471) and are located within the cluster tidal radius. The sources V1 and V2 are putative cluster members from Mochejska et al. (2000), but V2 has no proper motions in UCAC4 and V1 has rather incompatible proper motions, $(0.7 \pm 5.7,22.2 \pm 5.7)$ mas $_{\mathrm{yr}^{-1}}$.

\section{Polar paths}

Most globular clusters were born with the Galaxy, but some others were accreted. Each Galactic globular cluster and satellite

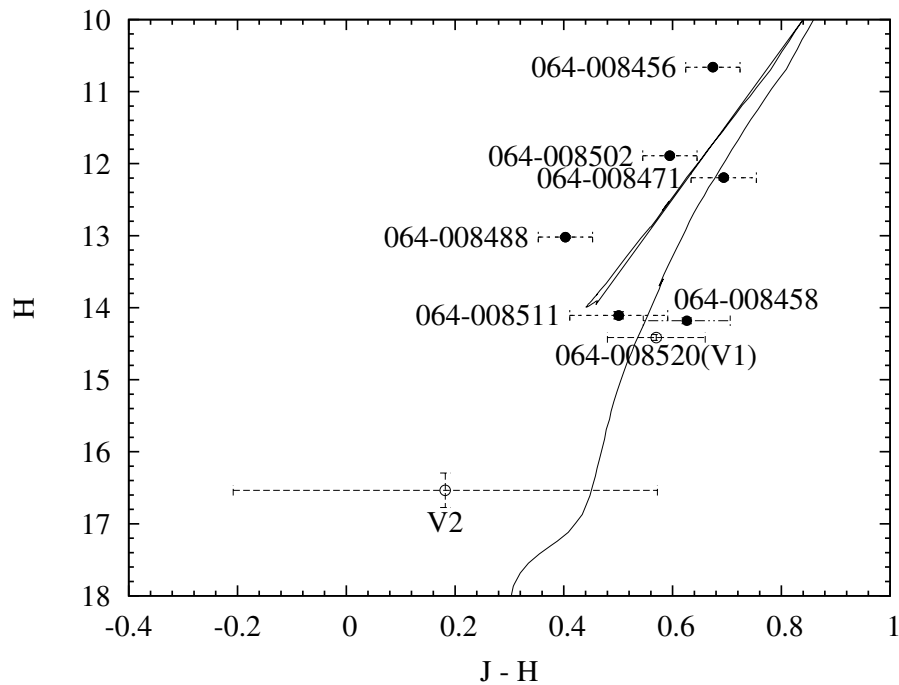

Fig. 5. 2MASS near-infrared CMD of stars within 8,5 from the cluster centre and with proper motions within $2 \sigma$ of our estimate for the cluster (black circles, labels are the UCAC4 designations). Variable stars V1 and V2 from Mochejska et al. (2000) are also indicated (empty circles).

dwarf galaxy orbits the Galaxy in a plane including the object's present position and the Galactic centre. This reference plane can be defined as the instantaneous or osculating orbital plane of the object. Following Lynden-Bell \& Lynden-Bell (1995) we consider the geometric loci of all possible normals to the galactocentric radius-vector for a particular object (see Appendix A for mathematical details). This defines the set of possible orbital poles for that object, globular cluster, or dwarf galaxy.

For E 3, the usual heliocentric galactic coordinates $(l, b)=$ $\left(292^{\circ} .270,-19.020\right)$ translate into galactocentric galactic coordinates $\left(l_{\mathrm{g}}, b_{\mathrm{g}}\right)=(232.550,-16.474)$. We solved Eq. (A.5) using a Monte Carlo approach and evaluated the statistical significance of all the crossing points of polar path intersections with that of E3 using the Kulldorff statistics (Kulldorff 1997). All studies coincide in classifying E 3 as a metal-rich globular cluster. For this globular cluster subset the most relevant intersection $(180 \sigma)$ appears to be located at $X=-0.42, Y=0.50$ or $(l, b)=\left(310^{\circ},+35^{\circ}\right)$, see Fig. 6 . In these diagrams a separation of 0.1 is equivalent to $\sim 6^{\circ}$. The poles in Fig. 6 are all within $2^{\circ}$ of the average pole. Prospective members of this dynamical family of objects include, besides E 3, the second most massive Galactic globular cluster NGC 104 (47 Tucanae), NGC 6171 (M 107), and NGC 6362. Additional members may be Terzan 2 and NGC 6652. All of them have ages in the range $12.8 \pm 0.6 \mathrm{Gyr}$ and metallicity $[\mathrm{Fe} / \mathrm{H}]$ in the range $(-0.69,-1.02)$. If we compare the polar paths of this group of objects with those of objects commonly associated with the Sagittarius stream (see Figs. 6 and A.1), the dispersion of the poles is even smaller in this case.

\section{Discussion and conclusions}

Clearly, more observations are required to investigate this interesting cluster further. In particular, detailed spectroscopy of the brightest stars can provide important information on the actual metallicity of the cluster and its radial velocity. However, our preliminary spectroscopic study has revealed that this cluster is profusely contaminated with field stars. On the other hand, its low luminosity and sparse population make it a unique template for the study of the final evolutionary phases in the life of a star 
cluster. An extensive spectroscopic study can be used to measure the radial velocity dispersion that in turn can be utilised to constrain the dynamical state of this object. In a cluster with a high binary fraction like E 3 (e.g. Veronesi et al. 1996), binary orbital motions can mask small velocity dispersions. Multi-epoch observations can be used to separate single from binary systems. Unfortunately, E 3 is among the most elusive and challenging known globular clusters because field contamination severely hampers spectroscopic studies.

The globular cluster E 3 does not have a clear horizontal branch and that makes its distance and age determination rather uncertain. Our values differ significantly from those in Sarajedini et al. (2007), namely $8.1 \mathrm{kpc}$ and $10 \mathrm{Gyr}$, respectively. The value of $A_{V}$ used in this study is nearly $17 \%$ lower than those used in previous works. Our isochrone fitting indicates that E 3 is probably very old, with an age of about $13 \mathrm{Gyr}$ and its heliocentric distance is nearly $10 \mathrm{kpc}$. We have confirmed that it is also somewhat metal rich with $[\mathrm{Fe} / \mathrm{H}]=-0.7$. For its kinematics, our tentative estimate for the proper motion is $\left(\mu_{\alpha} \cos \delta, \mu_{\delta}\right)=(-7.0 \pm 0.8,3.5 \pm 0.3)$ mas $\mathrm{yr}^{-1}$. A tangential velocity of $382 \pm 79 \mathrm{~km} \mathrm{~s}^{-1}$ is derived from our determination of the cluster proper motion and a radial velocity of $45 \pm 5 \mathrm{~km} \mathrm{~s}^{-1}$ is obtained from our spectroscopy, in the solar rest frame. The E 3 stellar system is one of the most intriguing globular clusters in the Galaxy. Its old age and high metallicity is a peculiar combination, only seen in a handful of objects like the far more conspicuous NGC 104 (47 Tucanae) that may share a stellar stream with it.

Acknowledgements. We thank the anonymous referee for the constructive and useful report. C.M.B. acknowledges support from FONDECYT through regular project 1150060 . In preparation of this research note, we made use of the NASA Astrophysics Data System and the ASTRO-PH e-print server. This research has made use of the SIMBAD and VizieR databases operated at CDS, Strasbourg, France. This publication makes use of data products from the Two Micron All Sky Survey, which is a joint project of the University of Massachusetts and the
Infrared Processing and Analysis Center/California Institute of Technology, funded by the National Aeronautics and Space Administration and the National Science Foundation.

\section{References}

Ashman, K. M., \& Zepf, S. E. 2008, Globular Cluster Systems (Cambridge: Cambridge Univ. Press)

Baume, G., Carraro, G., Costa, E., Méndez, R. A., \& Girardi, L. 2007, MNRAS, 375, 1077

Bressan, A., Marigo, P., Girardi, L., et al. A. 2012, MNRAS, 427, 127

Carraro, G., \& Costa, E. 2007, A\&A, 464, 573

Carraro, G., Zinn, R., \& Moni Bidin, C. 2007, A\&A, 466, 181

Coelho, P., Barbuy, B., Meléndez, J., Schiavon, R. P., \& Castilho, B. V. 2005, A\&A, 443, 735

Forbes, D. A., \& Bridges, T. 2010, MNRAS, 404, 1203

Freeman, K., \& Bland-Hawthorn, J. 2002, ARA\&A, 40, 487

Fuhrmann, K., Axer, M., \& Gehren, T. 1994, A\&A, 285, 585

Gratton, R. G., \& Ortolani, S. 1987, A\&AS, 67, 373

Hanes, D. A. 1980, in Globular Clusters, eds. D. Hanes, \& B. Madore (Cambridge: Cambridge Univ. Press), 1

Harris, W. E. 1976, AJ, 81, 1095

Harris, W. E. 1996, AJ, 112, 1487

Harris, W. E. 2010, Catalog of parameters for Milky Way Globular Clusters: The Database, http://www.physics.mcmaster.ca/resources/globular. html

Kulldorff, M. 1997, Commun. Statist.: Theory Meth., 26, 1481

Lan, S.-H., Kong, A. K. H., Verbunt, F., et al. 2010, ApJ, 712, 380

Lauberts, A. 1976, A\&A, 52, 309

Lynden-Bell, D., \& Lynden-Bell, R. M. 1995, MNRAS, 275, 429

McDonald, I., Boyer, M. L., van Loon, J. T., et al. 2011, ApJS, 193, 23

Mochejska, B. J., Kaluzny, J., \& Thompson, I. 2000, Acta Astron., 50, 105

Moni Bidin, C., de la Fuente Marcos, R., de la Fuente Marcos, C., \& Carraro, G. 2010, A\&A, 510, A44

Sarajedini, A., Bedin, L. R., Chaboyer, B., et al. 2007, AJ, 133, 1658

Schlafly, E. F., \& Finkbeiner, D. P. 2011, ApJ, 737, 103

Stetson, P. B. 1994, PASP, 106, 250

Tonry, J., \& Davis, M. 1979, AJ, 84, 1511

van den Bergh, S., Demers, S., \& Kunkel, W. E. 1980, ApJ, 239, 112

Veronesi, C., Zaggia, S., Piotto, G., Ferraro, F. R., \& Bellazzini, M. 1996, in Formation of the Galactic Halo... Inside and Out, eds. H. Morrison, \& A. Sarajedini (San Francisco: ASP ), ASP Conf. Ser., 92, 301

Zacharias, N., Finch, C. T., Girard, T. M., et al. 2013, AJ, 145, 44

Page 6 is available in the electronic edition of the journal at http: //www . aanda. org 

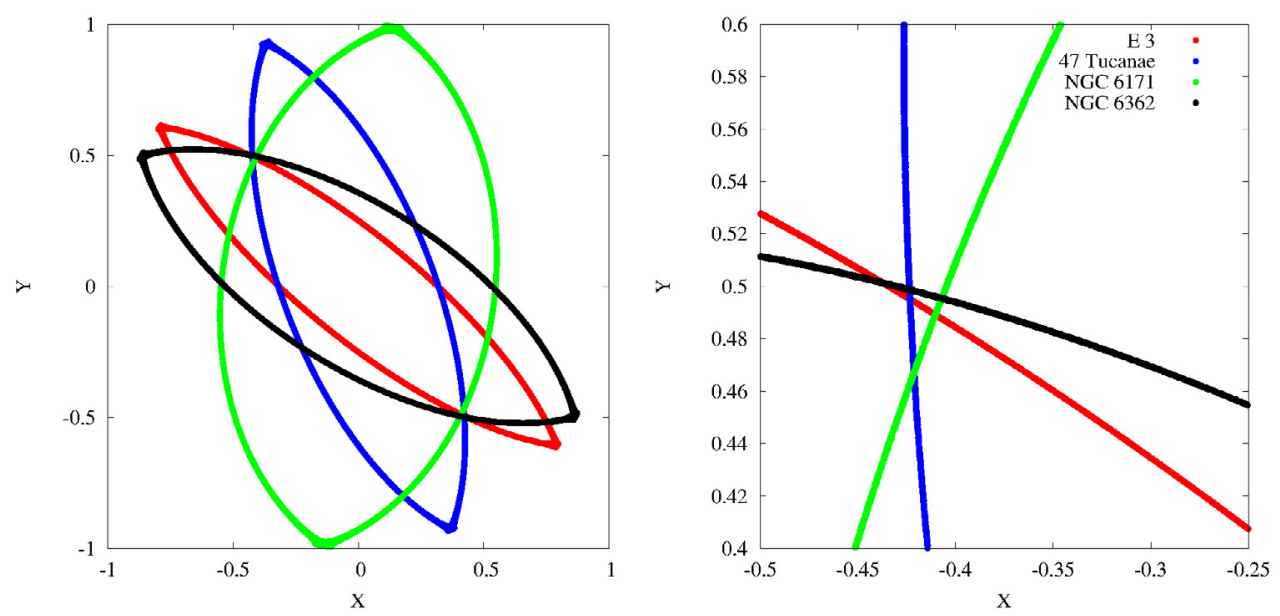

Fig. 6. Left panel: polar paths of objects believed to be part of the E 3 stream. Right panel: close-up.

\section{Appendix A: Polar path technique analysis}

Each Galactic globular cluster and satellite dwarf galaxy orbits the Galaxy in a plane including the object's present position and the Galactic centre. This reference plane can be defined as the instantaneous orbital plane of the object. Following Lynden-Bell $\&$ Lynden-Bell (1995) we consider the geometric loci of all possible normals to the galactocentric radius-vector for a particular object. This defines the set of possible orbital poles for that object, globular cluster, or dwarf galaxy. We consider a system of galactic coordinates on the unit sphere $\left(x^{2}+y^{2}+z^{2}=1\right)$ centred on the Galactic centre; the coordinates of any object in that system are given by

$$
(x, y, z)=\left(\cos l_{\mathrm{g}} \cos b_{\mathrm{g}}, \sin l_{\mathrm{g}} \cos b_{\mathrm{g}}, \sin b_{\mathrm{g}}\right),
$$

where $l_{\mathrm{g}}$ and $b_{\mathrm{g}}$ are the galactocentric (not the regular heliocentric) galactic longitude and latitude, respectively. The galactocentric radius-vectors for an object and its pole are perpendicular, therefore

$$
x \cos l_{\mathrm{g}} \cos b_{\mathrm{g}}+y \sin l_{\mathrm{g}} \cos b_{\mathrm{g}}+z \sin b_{\mathrm{g}}=0 .
$$

Finding $z(x, y)$ from Eq. (A.2), replacing it on the expression of the unit sphere, and reorganising we obtain

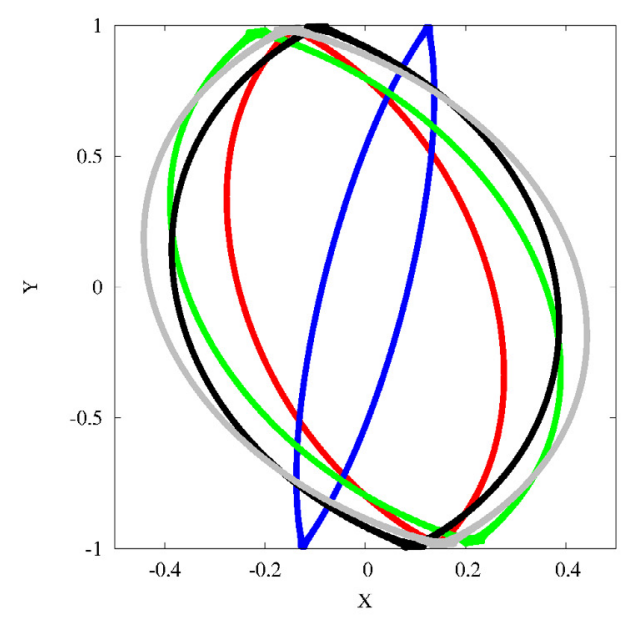

$x^{2}\left(1+\frac{\cos ^{2} l_{\mathrm{g}}}{\tan ^{2} b_{\mathrm{g}}}\right)+y^{2}\left(1+\frac{\sin ^{2} l_{\mathrm{g}}}{\tan ^{2} b_{\mathrm{g}}}\right)+2 x y \frac{\sin l_{\mathrm{g}} \cos l_{\mathrm{g}}}{\tan ^{2} b_{\mathrm{g}}}=1$.

If we now consider the Lambert azimuthal equal-area projection given by the expressions

$(X, Y)=(x / \sqrt{1+z}, y / \sqrt{1+z})$,

with $z=1-X^{2}-Y^{2}$, Eq. (A.3) can be rewritten as

$\left(2-X^{2}-Y^{2}\right)\left(X^{2}+Y^{2}+\left(X \cos l_{\mathrm{g}}+Y \sin l_{\mathrm{g}}\right)^{2} \frac{1}{\tan ^{2} b_{\mathrm{g}}}\right)=1$.

This expression provides the positions of all the possible poles associated with the object. The intersection (if any) of polar paths gives the putative pole of a group of (perhaps) dynamically related objects. Once the pole has been found, its position on the sky relative to the Galactic centre is given by $\left(l_{P}, b_{P}\right)=\left(\arctan \left(Y_{P} / X_{P}\right), \arcsin \left(1-X_{P}^{2}-Y_{P}^{2}\right)\right)$, see Eqs. (A.1) and (A.4). Polar paths of objects possibly associated with E3 are shown in Fig. 6. For comparison, those of objects widely regarded as part of the Sagittarius stream, streams $8 \mathrm{a}$ and $8 \mathrm{~b}$ in Table 2 of Lynden-Bell \& Lynden-Bell (1995), are plotted in Fig. A.1.

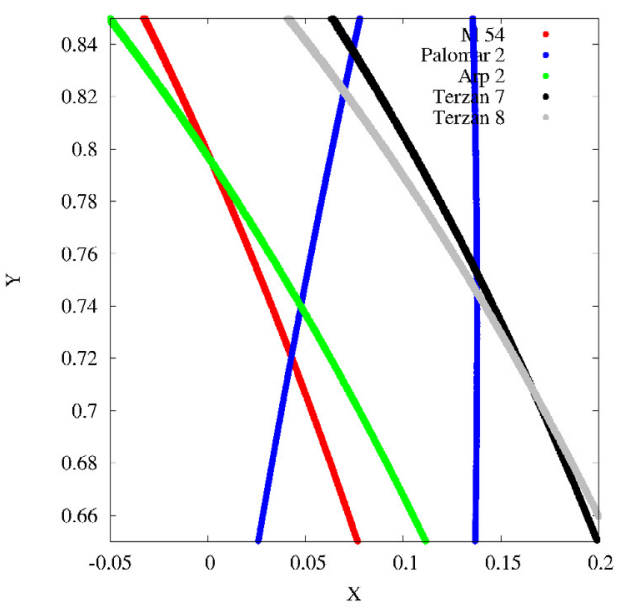

Fig. A.1. Left panel: polar paths of objects believed to be part of the Sagittarius stream, streams 8a and 8b in Table 2 of Lynden-Bell \& Lynden-Bell (1995). Right panel: close-up. 